\title{
Bilateral Endoscopic Total Extraperitoneal (TEP) Inguinal Hernia Repair Does not Induce Obstructive Azoospermia: Data of a Retrospective and Prospective Trial
}

\author{
Markus Schäfer
}

Published online: 29 April 2011

(c) Société Internationale de Chirurgie 2011

\section{Introduction}

In this issue of the journal, the clinical study by Skawran et al. [1] that meticulously assessed the risk of infertility, i.e., the mesh-related obstruction of the cord structures, after endoscopic bilateral inguinal hernia repair is presented. The authors investigated 59 male patients by using testicular ultrasound, levels of sexual hormones, and repeated spermiograms. Since none of the analyzed parameters changed, the authors concluded that preperitoneal mesh placement during endoscopic bilateral hernia repair has no impact on postoperative male fertility.

The wide use of mesh for inguinal hernia repair in the Western world has significantly lowered recurrence rates. Thus, other complications are coming into the focus, particularly the adverse effects of implanted mesh. The local interaction of mesh and normal body tissues is assumed to be the pathophysiological basis for mesh-related complications. However, dissection and handling of tissues to prepare the field for mesh insertion also play pivotal roles. The sensitivities of nerves, vessels, the spermatic cord, and fascia to mechanical stress are different. Once in situ, the physicochemical properties of the different mesh types largely determine local inflammatory reaction and subsequent mesh integration.

Testicular lesions and spermatic cord injuries are rare complications of hernia surgery and reported incidences

\footnotetext{
M. Schäfer

Department of Visceral Surgery, University Hospital

of Lausanne, Lausanne, Switzerland

M. Schäfer ( $\square)$

CHUV, Centre Hospitalier Universitaire Vaudois, Rue du

Bugnon 46, CH-1011 Lausanne, Switzerland

e-mail: markus.schaefer@access.unizh.ch
}

are below $1 \%$ [2]. Nevertheless, case reports evoked the potential risk of infertility due to an obstructive azoospermia, and animal studies seemed to provide some evidence of such a complication [1]. But how common is such problem in clinical practice and how significant is it? Our answer is that there is no such problem, and the issue is greatly overestimated. Why?

First, the use of mesh has not changed the incidence of urological complications after inguinal hernia repair during the past few decades. Indeed, a relevant increase of such a delicate problem would have been detected and published. In particular, manufacturers would have been alerted of the problem and would be fearful of legal and financial consequences. From a purely mechanical point of view, it seems to be very unlikely that a piece of mesh would cause a luminal obstruction, that is if the spermatic cord is not injured during dissection.

Second, mesh technology has evolved. The former heavy-weight meshes have been replaced by light-weight, partially absorbable or coated meshes. Also, surgeons are now much more experienced with safely implanting the mesh.

Third, animal models do not adequately reflect the clinical situation in humans. In small animals in particular, cord structures are much thinner and therefore more vulnerable to any manipulation [3, 4].

Fourth, the study by Skawran et al. [1] provides good evidence that the problem of male infertility due to obstruction of the spermatic cord has no clinical importance. Moreover, $90 \%$ of all inguinal hernia repairs are unilateral operations and fertility is preserved by the nonoperated side. As a consequence, no further or larger trials are needed.

And last but not least, the mean age of male patients undergoing inguinal hernia repair in most series ranges 
from 40 to 55 years $[1,5,6]$. High sexual activity combined with the strong wish to make a baby at $50+$ years has been hyped by the media, which provide us with news about celebrities having children at that age. However, there is a distinct difference between a lifestyle suggested by the media and the reality for most of us.

This last issue raises the interesting question: How do we look at complications? In the good old days, when patients respectfully received any verdict from their doctors, complications were accepted as inevitable sequels of medical treatment. Nobody would have even thought of talking about postoperative infertility. Nowadays, we believe in the new paradigm of an everlasting youth and the role of modern medicine is to help attain and preserve it. Complications such as mesh-related infertility are artificially hyped because they could threaten a man's image. In reality, good evidence to support such a hypothesis is lacking. It is highly questionable whether obstructive azoospermia after inguinal hernia mesh repair exists, but even if it did occur, it is not a real problem for 50-year-old men. We are biased by modern lifestyle and are victims of the great success of modern surgery.

\section{References}

1. Skawran S, Weyhe D, Belyaev O, Bauer KH (2011) Bilateral endoscopic total extraperitoneal (TEP) inguinal hernia repair does not induce obstructive azoospermia: data of a retrospective and prospective trial. World J Surg. doi:10.1007/s00268-011-1072-0

2. Markar SR, Karthikesalingam A, Alam F et al (2010) Partially or complete absorbable versus nonabsorbable mesh repair for inguinal hernia. Surg Laparosc Endosc Percutan Tech 20:213-219

3. Berndsen FH, Bjursten LM, Simanaitis M et al (2004) Does mesh implantation affect the spermatic cord structures after inguinal hernia surgery? Eur Surg Res 36:318-322

4. Uzzo RG, Lemack GE, Morrissey KP et al (1999) The effects of mesh bioprosthesis on the spermatic cord structures: A preliminary report in a canine model. J Urol 161:1344-1349

5. Shah NR, Mikami DJ, Cook C et al (2011) A comparison of outcomes between open and laparoscopic surgical repair of recurrent inguinal hernias. Surg Endosc. doi:10.1007/s00464010-1564-2

6. Perko Z, Rakić M, Pogorelić Z et al (2011) Laparoscopic transabdominal preperitoneal approach for inguinal hernia repair: a five-year experience at a single center. Surg Today 41:216-221 